\title{
CORRIGENDUM TO "A ZERO DENSITY ESTIMATE FOR DEDEKIND ZETA FUNCTIONS OF PURE EXTENSION FIELDS"
}

\author{
By
}

\author{
Koichi KAWADA
}

The introduction of the recent article [1] contains an erroneous account on the reducibility of the polynomial $x^{k}-n$, and the following description should be substituted for the last paragraph on p. 357 of [1]:

"Let $\mathscr{I}_{k}$ be the set of all the integers $n$ such that $x^{k}-n$ is irreducible in $Q[x]$. One may show that if $x^{k}-n$ is reducible then $|n|$ is a $p$-th power of an integer for some $p \mid k$, namely,

$$
\mathscr{I}_{k} \supset \mathbb{Z} \backslash\left(\bigcup_{p \mid k} \mathbb{Z}^{p}\right),
$$

where $\mathbb{Z}$ is the integer ring, and $\mathbb{Z}^{p}$ denotes the set of all the integers of the form $\pm m^{p}$ with $m \in \mathbb{Z}$."

This change does not influence the central argument of [1] at all, and its body remains valid.

The author is deeply obliged to Professor Gerhard Turnwald for his indication on the above matter, and for the following information (see Ch. VI, §9, Theorem 9.1 of Lang [2]). When $4 \nmid k$, the polynomial $x^{k}-n$ is reducible if, and only if, $n$ is a $p$-th power of an integer for some prime $p \mid k$. When $4 \mid k$, however, it is reducible if, and only if, $n$ is a $p$-th power of an integer for some $p \mid k$, or $n$ is of the form $-4 m^{4}$ with an integer $m$. Therefore the relation (1) in [1] is correct only when $4 \nmid k$, and fails to hold when $4 \mid k$.

\section{References}

[1] Kawada, K., A zero density estimate for Dedekind zeta functions of pure extension fields, Tsukuba J. Math. 22 (1998), 357-369.

[2] Lang, S., "Algebra." 3rd ed. Addison-Wesley, New York, 1993.

Department of Mathematics

Faculty of Education

Iwate University

Morioka, 020-8550 Japan

E-mail: kawada@iwate-u.ac.jp 